ISSN 1991-8631

Original Paper

http://indexmedicus.afro.who.int

\title{
Perceptions paysannes et impacts des changements climatiques sur la production et la diversité variétale de l'igname dans la zone aride du nord-ouest du Bénin
}

\author{
Y. L. LOKO ${ }^{1}$, A. DANSI ${ }^{1 *}$, A. P. AGRE ${ }^{1}$, N. AKPA ${ }^{1}$, I. DOSSOU-AMINON ${ }^{1}$, \\ P. ASSOGBA ${ }^{1}$, M. DANSI $^{1}$, K. AKPAGANA ${ }^{2}$ et A. SANNI ${ }^{3}$ \\ ${ }^{1}$ Laboratoire de Biotechnologie, Ressources Génétiques et Amélioration des Espèces Animales et Végétales \\ (BIORAVE), Faculté des Sciences et Techniques de Dassa, Université d'Abomey-Calavi, BP 526 Cotonou, \\ Benin. \\ ${ }^{2}$ Laboratoire de Botanique, Faculté des sciences (FS), Université de Lomé, BP 1515, Lomé, Togo. \\ ${ }^{3}$ Laboratory of Biochemistry and Molecular Biology, Faculty of Sciences and Technology (FAST), \\ University of Abomey-Calavi (UAC), P.O. Box 526 Cotonou, Benin. \\ *Auteur correspondent, E-mail: adansi2001@gmail.com ; Tel : +22997276598
}

\section{RESUME}

L'igname (Dioscorea spp.) est une culture vivrière de grande importance pour la sécurité alimentaire au Bénin. Cependant, sa production dans le département de l'Atakora (extrême Nord-Ouest) est sérieusement affectée par les changements climatiques. Pour analyser les perceptions paysannes des changements climatiques, documenter les impacts de ceux-ci sur la production des ignames et les stratégies d'adaptation développées pour y faire face, quinze (15) villages ont été sélectionnés au hasard et explorés. Les producteurs ont signalé des fluctuations de température et de pluviométrie, une augmentation de la température et une diminution de la pluviométrie corroborées par l'analyse des données météorologiques. L'âge et le genre influencent significativement la perception paysanne des changements climatiques. Les impacts les plus importants sont la baisse de rendement, la pourriture des tubercules et des semences, l'appauvrissement et la dégradation des sols et la prolifération des insectes et ravageurs. Les stratégies adoptées inclus entre autres le paillage des buttes, le déplacement vers les zones humides et l'utilisation de bonnes pratiques culturales et de la diversité variétale. Cette capacité d'adaptation des producteurs pourrait être accompagnée par les politiques publiques mais ceux-ci devront tenir compte des différents facteurs influençant la capacité d'adaptation des producteurs déterminés au cours de cette étude.

(C) 2013 International Formulae Group. All rights reserved.

Mots clés : Critères de préférence, cultivars tolérants, sécheresse, stratégies d'adaptation, variabilité climatique.

\section{INTRODUCTION}

L'agriculture constitue l'un des secteurs les plus importants de l'économie béninoise et implique la majorité de la population (Yabi et Afouda, 2012). Ce secteur est particulièrement important en termes d'emplois $(48 \%$ de la population du pays) et de contribution au produit intérieur brut $(45 \%$ de la structure du PIB) et fournit $88 \%$ des recettes d'exportation du pays (Labintan et Ding, 2012). En dépit de 
sa forte contribution à l'économie dans son ensemble, ce secteur est confronté à de nombreuses contraintes dont les changements climatiques (Yabi et Afouda, 2012). En effet, le Bénin connaît depuis plus de 40 ans de fortes variabilités climatiques caractérisées par une fluctuation de la période et de la durée des précipitations, une variation de la pluviométrie annuelle, un climat de plus en plus chaud, la sécheresse, la dégradation des sols, des inondations inattendues, des vents violents et la prolifération des maladies et ravageurs (Yabi et Afouda, 2012). Ces changements climatiques, grande source d'inquiétude pour les agriculteurs, affectent négativement les rendements des cultures par leurs impacts sur la croissance et le développement des plantes et sur la diversité variétale (Luka et Yahaya, 2012; Adesiji et al., 2012). Cette baisse des rendements agricoles en raison des mauvaises conditions pédoclimatiques conduit à coup sûr à l'insécurité alimentaire croissante, à la vulnérabilité des communautés agricoles, à la réduction des revenus des ménages et à une augmentation de la pauvreté (Srivastava et al., 2012).

Parmi les principales cultures vivrières produites au Bénin, l'igname (Dioscorea spp) est l'une des plus importantes (Loko et al., 2013) et constitue l'aliment de base et une source importante de revenus pour plus de $75 \%$ de la population rurale repartie du nord au sud sur plus de $60 \%$ de la superficie nationale (Loko et al., 2013). Sa production nationale annuelle est en moyenne de $2366000 \mathrm{~T}$ (Loko et al., 2013). Deux espèces majeures (Dioscorea alata; complexe Dioscorea cayenensis- Dioscorea rotundata) sont cultivées (Mignouna et Dansi, 2003). Parmi celles-ci, le complexe D. cayenensis- D. rotundata est le plus cultivé (plus de $95 \%$ des superficies), le plus répandu, le plus diversifié et le plus consommé (Loko et al., 2013). Au Bénin comme dans d'autres pays de l'Afrique de l'ouest, l'igname joue un rôle primordial dans la sécurité alimentaire mais sa production est malheureusement sujette à de nombreuses contraintes environnementales (biotiques et abiotiques) et agronomiques d'importances variables selon les zones agroécologiques (Dansi et al., 2013). Dans le nord-ouest (Département de l'Atacora) les variabilités climatiques surtout marquées par la sécheresse de plus en plus importante et l'inadaptabilité des variétés locales cultivées aux facteurs pédoclimatiques actuels sont, selon les producteurs, les contraintes les plus importantes (Loko et al., 2013). La sensibilité de la production d'igname aux principaux paramètres climatiques que sont les précipitations, la température, la lumière et la photopériode la rende donc vulnérable aux changements climatiques et conduisent à une baisse importante du rendement (Sadauki et Olanrewaju, 2012). Pour faire face à cette situation et réduire les impacts négatifs des changements climatiques les producteurs ont développé de nombreuses stratégies qu'il est important de documenter pour évaluation et valorisation (Tidjani et Akponikpe, 2012; Naess, 2013).

L'adaptation aux changements climatiques est un processus qui requiert initialement que les producteurs perçoivent que le climat a changé et alors identifient les adaptations utiles à mettre à exécution (Moustapha et al., 2012). Ceux-ci, en contact direct avec les éléments de la nature dans leur environnement physique et biologique (Tidjani et Akponikpe, 2012) jouent un rôle actif dans le développement de technologies permettant de résoudre efficacement leurs problèmes (Naess, 2013). Les populations rurales du Bénin ont sans doute une certaine perception de l'évolution du climat en fonction de laquelle, elles ont toujours développées des stratégies d'adaptation, générant ainsi au fil des années des connaissances et un savoir-faire jugés globalement pertinents (Tidjani et Akponikpe, 2012). Afin d'améliorer les politiques visant à promouvoir des stratégies d'adaptation adéquates aux changements climatiques pour la production de l'igname, une meilleure compréhension de la façon dont ils perçoivent 
les changements climatiques et les mesurent, des facteurs déterminants la perception des changements climatiques par les producteurs et des facteurs qui influent sur la décision des producteurs à adapter de nouvelles pratiques agricoles est nécessaire (Tazeze et al., 2012). La présente étude effectuée dans le département de l'Atakora au nord-ouest du Bénin vise à:

- Documenter les perceptions paysannes des changements climatiques et les comparer aux données météorologiques des quarante dernières années ;

- Identifier les facteurs influençant la perception des changements climatiques par les producteurs ainsi que les impacts de la variabilité climatique sur la production et la diversité variétale de l'igname ;

- Identifier les stratégies d'adaptation développées par les producteurs d'igname visà-vis des changements climatiques et les déterminants des méthodes d'adaptation employés par les producteurs d'igname ;

- Identifier dans cette zone d'étude les critères paysans de préférence variétale pour les besoins de la sélection et de la vulgarisation.

\section{MATERIEL ET METHODES}

\section{Présentation de la zone d'étude}

L'étude a été conduite dans le département de l'Atakora situé au nord-ouest du Bénin (Figure 1). Ce département est localisé dans une zone agroécologique semiaride, caractérisée par une pluviométrie imprévisible et irrégulière (800 - $950 \mathrm{~mm} / \mathrm{an})$ avec seulement une saison pluvieuse et une saison sèche qui dure le plus souvent plus de cinq mois (Dansi et al., 2010). La région est montagneuse avec comme principales formations pédologiques des sols ferrugineux et des bas-fonds (Dansi et al., 2010). La zone d'étude est subdivisée en six communes (Boukoumbé, Cobli, Materi, Natitingou, Tanguiéta et Toukountouna) habitée par cinq groupes ethniques que sont: Berba, Bialli, Ditamari, Natimba et Wama. La population est essentiellement rurale et l'agriculture est la principale activité (Dansi et al., 2010). Pour les enquêtes et dans le souci d'obtenir une bonne couverture de la zone d'étude et de prendre en compte les zones marginales de production (Dansi et al., 2010), quinze villages ont été sélectionnés au hasard à travers les différentes communes et aires ethniques (Figure 1).

Dans chacun des villages retenus, les données ont été collectées en utilisant les outils (questionnaires) et méthodes (enquête de groupe, enquête individuelle, visite de champs) de la recherche participative (Kombo et al. 2012). Par village, le groupe de producteurs enquêtés est constitué de 20 à 30 producteurs d'igname des deux sexes et de différents âges (vieux, jeunes) identifiés et rassemblés avec l'aide du chef de village et des responsables des associations des producteurs. Du fait de la diversité ethnique, des interprètes ont été localement recrutés pour faciliter les discussions et les échanges avec les producteurs selon Kombo et al. (2012). L'enquête de groupe a été une approche clé pour générer des informations sur la perception des producteurs sur les changements climatiques, les événements climatiques majeurs ayant marqué la vie de leur village et les stratégies d'adaptations communautaires développées (Tidjani et Akponikpe, 2012).

L'enquête individuelle a concerné dix producteurs (chefs de ménage ou leurs femmes ayant au moins 10 ans d'expérience dans la production d'igname) pris dans dix ménages sélectionnés dans chacun des villages enquêtés selon la méthode de transect décrite par Dansi et al. (2010). Les données sont collectées sur la base de questionnaires selon Nyanga et al. (2011). Celles-ci ont trait aux caractéristiques sociodémographiques des ménages enquêtés (sexe, âge, taille du ménage, niveau d'éducation, années d'expérience dans la production de l'igname, main d'œuvre employée, taille des exploitations et appartenance ou non à une association paysanne), aux perceptions paysannes des changements climatiques 
(pluviométrie, température, humidité relative, vent violents, etc.) ces 40 dernières années, à l'impact des changements climatiques sur la production et la diversité variétale de l'igname, aux stratégies mises en place par les producteurs pour minimiser les risques et adapter leur production d'igname aux nouvelles conditions environnementales, et aux critères paysans de préférence variétale.

Pour des nécessités de comparaison entre déclarations paysannes et informations scientifiques, les données climatologiques (température $\left({ }^{\circ} \mathrm{C}\right)$, humidité relative $(\%)$, pluviométrie $(\mathrm{mm})$ et nombre de jours de pluie) recueillies dans la zone d'étude ces 40 dernières années (1971 à 2010) ont été collectées à partir de l'ASECNA (Agence pour la Sécurité et la Navigation Aérienne).

\section{Analyses statistiques}

Les données collectées ont été analysées par la statistique descriptive (moyenne, écart-type, variance) et la statistique différentielle avec les logiciels Minitab version 14.0 et Stata SE version 10.0 et les résultats présentées sous forme de tableaux et de figures selon Kisauzi et al. (2012). Pour analyser les relations entre les 40 différentes années considérées, celles-ci ont été prises comme individus et les données climatiques (pluviométrie annuelle, température, humidité relative) qui les caractérisent comme variables et utilisées dans une analyse en composante principale (ACP) avec le logiciel Minitab version 14.0 selon Uguru et al. (2011). L'identification des années ayant eu des conditions climatiques extrêmes a été faite en calculant le pourcentage du déficit ou de l'excédent des précipitations par rapport à la moyenne selon Yabi et Afouda (2012). Les déterminants qui influent sur la perception paysanne des changements climatiques ont été analysés par la régression logique binaire (Oyekale et Oladele, 2012) selon le model suivant :

$\mathbf{y i}=\mathbf{x i} \boldsymbol{\beta}+\mathbf{\varepsilon i}$

Où yi est la variable latente indiquant si oui ou non le producteur d'igname perçoit les changements climatiques, xi est l'ensemble des variables explicatives indiquant les facteurs qui influencent la perception paysanne des changements climatiques, $\mathbf{\varepsilon i}$ est l'erreur standard. La variable dépendante est une variable fictive égale à 1 si le producteur perçoit les changements climatiques et 0 au cas contraire (Oyekale et Oladele, 2012). Les variables explicatives considérées sont parmi celles signalées par différents auteurs (Oyekale et Oladele, 2012) comme affectant la prise de conscience des changements climatiques par les producteurs. La régression logique binaire a été aussi utilisée selon Nabikolo et al. (2012) pour déterminer les facteurs qui influencent la décision des producteurs d'igname à développer des stratégies d'adaptation. Dans ce cas yi représente dans l'équation (1) une variable dépendante dichotomique (la variable prend la valeur 1 si le producteur adopte une stratégie d'adaptation en réponse aux changements climatiques perçus et la valeur 0 dans le cas contraire) et xi est l'ensemble des variables explicatives.

Les facteurs influençant le choix des producteurs d'igname à utiliser une méthode particulière d'adaptation face aux changements climatiques ont été déterminés en utilisant le modèle logit multinomial selon Tazeze et al. (2012). Dans ce modèle, la variable dépendante est multinomiale avec autant de catégories que le nombre de méthodes d'adaptation aux changements climatiques inventoriées dans la zone d'étude. La spécification du modèle empirique ou sous forme réduite se présente comme suit (Tazeze et al. 2012):

\section{yi $=\mathbf{f}(\mathrm{X1}, \mathrm{X2}, \ldots, \mathrm{X7})$}

Où yi, variable dépendante polychotomique est la méthode d'adaptation choisie par le producteur d'igname et X1 à X7 sont les variables explicatives. Sur la base des informations recueillies sur les stratégies d'adaptations développées par les producteurs dans la zone d'étude, la variable dépendante (yi) est codée 1 pour «aucune adaptation », 2 pour « utilisation de la diversité variétale », 3 
pour «bonnes pratiques culturales (paillage et tuteurage) », 4 pour «drainage », 5 pour «changement de terrain» et 6 pour «multiples stratégies d'adaptation ». Les variables explicatives comprennent: $\mathrm{X} 1=$ niveau d'éducation, $\mathrm{X} 2$ = âge, $\mathrm{X} 3$ = sexe, $\mathrm{X} 4$ $=$ années d'expérience dans la production d'igname, $\mathrm{X} 5$ = taille de l'exploitation, $\mathrm{X} 6=$ main d'œuvre utilisée et X7 = appartenance à une association paysanne. La description détaillée des relations hypothétiques entre ces variables et l'adaptation aux changements climatiques est exposée dans Tazeze et al. (2012). L'estimation du modèle de la régression logique multinomiale a été faite par la normalisation d'une catégorie, définie comme «catégorie de base» ou «état de référence» (Tazeze et al., 2012). Dans cette étude, la catégorie «aucune adaptation»a été utilisée comme catégorie de base.

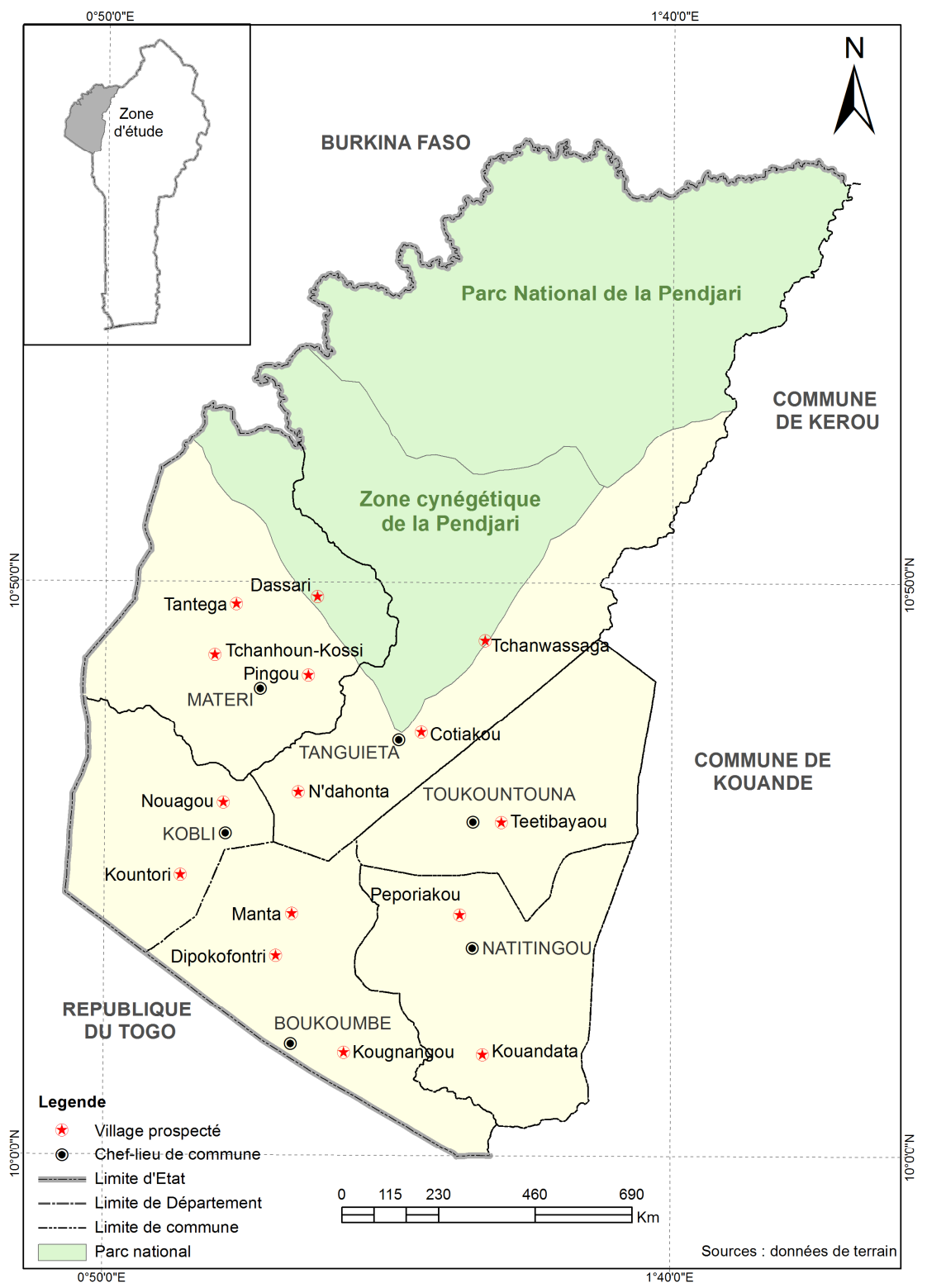

Figure 1: Carte du Nord-Ouest du Bénin montrant la localisation géographique des villages enquêtés. 


\section{RESULTATS}

\section{Caractéristiques sociodémographiques des} producteurs enquêtés

Les producteurs enquêtés étaient en majorité des hommes $(92,67 \%)$. Leurs âges variaient de 23 à 72 ans (47 ans en moyenne) et ils étaient à $58 \%$ des analphabètes. La majorité $(60.67 \%)$ des producteurs avait 41 à 60 ans d'âge. Plus de la moitié $(53,33 \%)$ des producteurs enquêtés ont 10 à 25 ans d'expérience dans la production d'igname et seulement $40,67 \%$ d'enquêtés avaient entre 26 à 45 ans d'expérience. La taille des ménages est de 10 en moyenne et celle des exploitations variait de 0,12 à 2,5 ha $(0,65$ ha en moyenne). Très peu de producteurs (20\%) sont membres d'une association paysanne.

Perceptions paysannes des changements climatiques et comparaison avec les données météorologiques

D'une manière générale, les producteurs enquêtés ont indiqué qu'une bonne pluviométrie ( $86 \%$ de réponses), une température moyenne (9,33\% de réponses), un vent doux $(2,67 \%$ de réponses $)$ et un ensoleillement moyen (2\% de réponses) sont les conditions idéales pour la croissance et le développement des plants et des tubercules d'igname. Ils ont indiqué $(98 \%$ des producteurs) que, pour la production de l'igname le climat dans le passé, caractérisé par des précipitations régulières $(67,33 \%$ de réponses), une température moyenne $(28,67 \%$ de réponses) et des vents moins violents (4\% de réponses) était meilleur au climat actuel et qu'il y a eu de façon notable des changements climatiques perceptibles surtout au niveau de la pluviométrie $(94,66 \%$ de réponses) et de la température (92,66\% de réponses).

$\mathrm{Au}$ niveau de la pluviométrie, les variabilités sont signalées à sept niveaux (Figure 2) dont trois (retard de pluie, coupures précoces des pluies, diminution de la quantité des pluies) sont les plus importants et représentent à eux seuls 80,03\% des réponses. Les pluies excessives sont aussi rapportées mais restent la variabilité pluviométrique la moins signalée $(4,46 \%$ de réponses). Au niveau de la température, les producteurs ont signalé une augmentation de la température et de la durée d'insolation, un début précoce ou tardif du harmattan et une réduction de la fraicheur matinale nocturne (entre $2 \mathrm{~h}$ et $6 \mathrm{~h}$ du matin) pendant la période d'harmattan (Figure 3). L'élévation de la température et l'augmentation de la durée d'insolation sont les deux changements les plus signalés avec respectivement $50,16 \%$ et $42,76 \%$ de réponses (Figure 3). La majorité des producteurs (98,32\%) ont signalé des impacts négatifs sur la qualité des sols et plus particulièrement l'appauvrissement (84,09\% de réponses) et la dégradation $(15,91 \%$ de réponses) des sols.

Les données pluviométriques de la zone d'étude des 40 dernières années (1971 2010) font apparaitre des fluctuations évidentes et une tendance à la hausse au fil des ans confirmée par la droite de régression (Figure 4a). La quantité de pluie a été particulièrement faible $(<1000 \mathrm{~mm})$ en 1977 , en 1983 et en 2000. Au-delà de 2007, les précipitations annuelles ont été systématiquement plus élevées que la valeur moyenne globale $(1193 \mathrm{~mm})$ enregistrées sur toute la période considérée. Le nombre de jours de pluie a connu aussi de nombreuses fluctuations mais avec une tendance générale à la baisse indiquée par la droite de régression linéaire (Figure 4b).

L'analyse des données climatiques relatives à la moyenne annuelle de la température enregistrée dans les stations météorologiques de la zone d'étude entre 1960 et 2010 montrent beaucoup de fluctuations et une tendance à la hausse soutenue par les droites de régression linéaire aussi bien au niveau des températures minimales qu'au niveau des températures maximales (Figure 5a). La température maximale moyenne 
annuelle la plus élevée $\left(34,4{ }^{\circ} \mathrm{C}\right)$ a été enregistrée en 2010 et la température minimale moyenne annuelle $\left(32{ }^{\circ} \mathrm{C}\right)$ a été enregistrée en 1962 (Figure 5a). La distribution intra-annuelle de la température (Figure 5b) montre que la dernière décennie (2000-2010) a été nettement plus chaude que les décennies précédentes.

La comparaison entre les données météorologiques et les perceptions paysannes a montré une similarité puisque les producteurs ont bien rapporté une augmentation de la température, une diminution du nombre de jours de pluie et aussi bien une baisse qu'une hausse de la pluviométrie.

Au cours des enquêtes, les producteurs ont mentionné dans différents villages des années dominées par des conditions climatiques extrêmes (sécheresse, inondations, vents violents). Les incidents majeurs d'inondations auraient eu lieu en 2003 et entre 2005 et 2010 alors que les cas de sécheresse extrême ont été signalés comme ayant eu lieu entre 1982 et 1987 et entre 1997 et 2009 dans presque tous les villages prospectés. Des vents violents auraient soufflé aussi bien au cours des saisons sèches et pluvieuses de 1993 et d'entre 2003 et 2009 à l'exception des années 2004 et 2008 dans des villages comme Nouagou et Kountori. La superposition des données pluviométriques avec les indications paysannes des années dominées par des conditions climatiques extrêmes a révélé de nombreuses concordances. Contrairement aux années 1986, 2004, 2008 et 2009, les années 1982, 1983,1984, 1997,1999, 2000, 2002 et 2006 signalées comme années de sécheresse extrême ont effectivement présenté selon les données météorologiques, une pluviométrie très inférieure à la moyenne $(1193 \mathrm{~mm})$ de la zone d'étude indiquant un déficit. Le taux de concordance entre la perception paysanne des années de sécheresse et les données météorologiques est de 66,67\%. Quant à la perception paysanne des années d'inondation le taux de concordance avec les données météorologiques est de $83,33 \%$ car à l'exception de l'année 2005 qui a présenté une hauteur annuelle pluviométrique inférieure à la moyenne, toutes les autres années signalées ont présenté un excès pluviométrique. D'une manière générale, les producteurs dans la zone d'étude semblent ne se rappeler que des événements climatiques extrêmes récents (moins de 30 ans ; 1982 à 2010).

L'analyse en composantes principales (ACP) effectuée pour analyser les relations entre les différentes années a révélé deux composantes principales expliquant $89,1 \%$ des variations totales des précipitations annuelles, de la température et de l'humidité relative au cours des quarante dernières années dans la zone d'étude (Figure 6). L'ACP a aussi mis en exergue des groupes d'années (marqués en couleurs sur la Figure 6) qui partagent ensemble les mêmes caractéristiques comme les années 1998, 2003, 2004, 2007, 2008, 2009, 2010 qui forment le groupe des années les plus pluvieuses et les années 1983, 1987, 1990, 2005, 2006 qui se dégagent comme ayant été les plus chaudes (Figure 6).

L'analyse de régression logique binaire (Tableau 1) a révélé que l'âge et le genre (sexe) sont, parmi les huit variables explicatives considérées, celles qui influencent de façon statistiquement significative les perceptions paysannes des changements climatiques (Table 1). Les producteurs ont mieux perçu les changements climatiques que les productrices et plus les enquêtés sont âgés mieux ils perçoivent les changements climatiques. La valeur Pseudo $\mathrm{R}^{2}$ de 0,1599 indique que $15 \%$ des variations de la perception des agriculteurs sont expliqués par les variables indépendantes incluses dans le modèle de régression binaire alors que la valeur du Chi-carré de 30,95 associé à la log-vraisemblance est hautement 
significative ( $p<0,0001)$ suggérant un fort pouvoir explicatif du modèle.

\section{Impacts des changements climatiques sur la production et la diversité variétale des ignames}

Dans la zone d'étude, les manifestations du changement climatique ont des impacts négatifs sur la production et la diversité variétale d'igname. Les impacts signalés par les producteurs sur la production de l'igname sont de quatre types : baisse de productivité $(41,35 \%$ de réponses), pourriture des semences sous terre $(20,67 \%$ de réponses), dessèchement suivi de la mort prématurée des plants (16,16\% de réponses), prolifération des maladies et des ravageurs et intensification de leurs attaques $(13,45 \%$ de réponses) et pourriture post récolte rapide des tubercules en stock $(8,37 \%$ de réponses). En groupe comme de façon individuelle, tous les producteurs ont rapporté l'abandon de nombreuses variétés d'igname du fait de leur inadaptabilités aux nouvelles conditions pédoclimatiques et donc des changements climatiques. Le nombre de cultivars déjà perdus ces vingt dernières années dans ce cadre a varié de 5 à 11 par village. Sous réserve de synonymie, 90 cultivars perdus ont été signalés à travers les quinze villages prospectés.

\section{Stratégies paysannes d'adaptation de la production d'igname aux impacts des changements climatiques}

Les enquêtes ont révélé que parmi les producteurs d'igname interrogés et qui ont reconnu l'existence de variabilité climatique, seulement $60,70 \%$ ont mis en place des stratégies d'adaptation qui varient avec les effets majeurs des changements climatiques signalés que sont la sécheresse, l'inondation, l'élévation de température et les vents violents.
Par rapport à la sécheresse sept stratégies sont utilisées (Figure 7a) parmi lesquelles le déplacement des champs vers les périphéries des bas-fonds $(30,43 \%$ de réponses), l'utilisation de variétés précoces ( $26,08 \%$ de réponses) et le paillage des buttes $(26,08 \%$ de réponses) sont les plus importantes (Figure 7a). Des producteurs ont aussi signalé l'utilisation des variétés résistantes (4,35\% de réponses). Sous réserve de synonymie, six cultivars d'igname (Enonan, Paroyobou, Tchinguita, Tinonyaaté, Wonnifeena, Wonnisaabou) ont été listés à travers la zone d'étude, comme résistants à la sécheresse. Pour faire face à l'inondation quatre stratégies d'adaptation sont mises en place par les producteurs (Figure 7b). Parmi celles-ci la confection de grosses buttes $(43,48 \%$ de réponses) et l'utilisation des cultivars tolérantes à l'excès d'humidité (24,78\% de réponses) sont les plus utilisées et constituent à elles seules $68,26 \%$ des réponses (Figure $7 b)$. Le paillage $(73,97 \%$ de réponses) et dans une moindre mesure le stockage des tubercules et semences sous ombrage $(12,33$ de réponses) sont les deux stratégies les plus utilisées par les producteurs d'igname pour répondre à l'élévation de température (Figure 7c). Par rapport aux effets néfastes des vents violents les producteurs utilisent surtout comme méthode d'adaptation le tuteurage (avec des branches courtes et très ramifiées) des plants d'igname $(74,99 \%$ de réponses) et la reconfection des buttes d'igname détruites en partie par le vent $(14,29 \%$ de réponses) (Figure 7d). Pour maintenir la fertilité des sols, les producteurs utilise la jachère $(59,39 \%$ de réponses), la rotation avec les légumineuses $(22,03 \%$ de réponses), la fertilisation (9,69\% de réponses), l'association des cultures $(5,73 \%$ de réponses $)$, enfouissement des herbes lors des labours ( $2,64 \%$ de réponses), le déplacement vers les sols fertiles (2,2\% de réponses), la brulure des résidus de récolte $(0,88 \%$ de réponses $)$ et 
l'augmentation de la superficie emblavée $(0,44 \%$ de réponses). Globalement, les stratégies développées peuvent être classées en quatre catégories: bonnes pratiques culturales, utilisation de la diversité variétale d'igname existante, changement de terrain et drainage (confection de buttes dans le sens de l'écoulement de l'eau).

L'analyse de régression logique binaire (Tableau 2) a révélé que parmi les huit variables explicatives considérées, seuls l'âge et l'expérience des producteurs influencent de façon statistiquement significative leur capacité à développer des stratégies d'adaptation de la culture d'igname aux changements climatiques (Tableau 2). Les résultats indiquent que les producteurs d'igname les plus expérimentés et les producteurs les moins âgés sont plus aptes à définir des stratégies d'adaptation (Tableau 2).

L'analyse de régression logique multinomiale effectuée pour déterminer les facteurs influençant le choix des producteurs d'igname à utiliser une méthode particulière d'adaptation face aux changements climatiques a révélé (Tableau 3) que l'âge du producteur affecte de façon significative $(\mathrm{p}<0,01)$ mais négativement l'utilisation d'une combinaison de stratégies, que le sexe (genre) influence de façon positive $(\mathrm{p}<0,001)$ la décision des producteurs à utiliser les cultivars résistants de la diversité variétale existante comme mesure d'adaptation aux effets négatifs des changements climatiques. Le genre affecte aussi négativement $(\mathrm{p}<0,05)$ l'utilisation à la fois de plusieurs stratégies (Tableau 3). L'éducation, l'expérience et la main d'œuvre disponible sont aussi apparu comme des facteurs discriminants à divers niveaux (Tableau 3). Pour la plupart des méthodes d'adaptation, la taille du ménage et la superficie emblavée n'ont eu aucune influence. Les valeurs du chi-carré obtenues dans l'analyse sont statistiquement significatives $(p<0,05)$ indiquant que le modèle a produit un bon ajustement et qu'il a un fort pouvoir explicatif.

\section{Critères paysans de préférence variétale}

Seize critères de préférence ou de sélection variétale ont été identifiés à travers les villages et les aires ethniques prospectés (Tableau 4). Parmi ces critères, 01 (forte valeur marchande) est économique, 04 sont culinaires et technologiques, 05 sont agronomiques et 06 sont environnementaux. Les critères environnementaux $(42,39 \%$ des réponses) ont été les plus importants. Parmi ceux-ci la résistance à la sécheresse $(19,10 \%$ des réponses) et la résistance aux ravageurs et maladies $(13,43 \%$ de réponses) sont les plus déterminants. Cette tendance semble être générale pour toutes les aires ethniques sauf chez les Natimba où la tolérance à l'excès d'humidité a pris une part importante $(11,94 \%$ de réponses) et est classée en deuxième position (Tableau 4). Au niveau des critères agronomiques, la conservation post-récole est apparue comme le critère le plus important dans toutes les aires ethniques. La forte productivité qui est en deuxième position chez les Berba et les Ditamari est classé troisième chez les Bialli, les Natimba et les Wama (Tableau 4). La bonne qualité du pilé a été le critère technologique et culinaire le plus important dans la zone d'étude $(11,24 \%$ de réponses) et à travers les aires ethniques à l'exception des Natimba pour qui, la bonne qualité des cossettes est le deuxième critère le plus important. La forte valeur marchande notée chez les Berba, les Ditamari, les Natimba et les Wama n'a pas été signalée par les Bialli. 


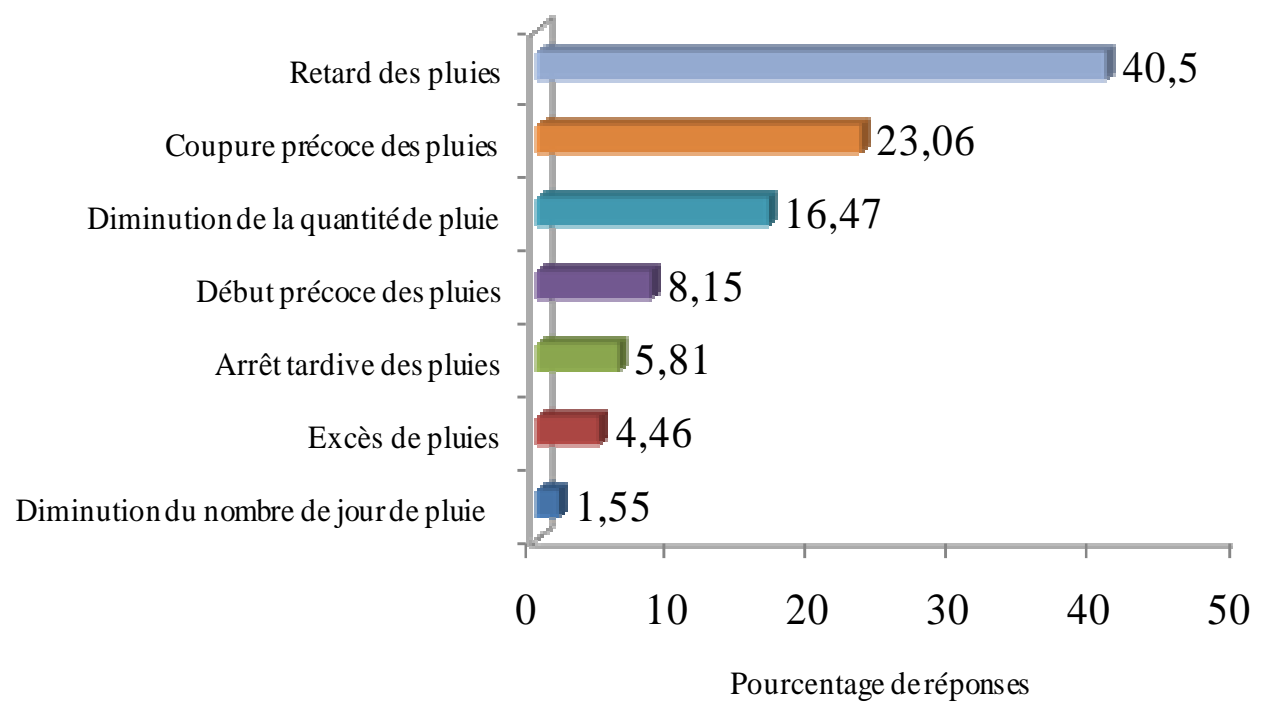

Figure 2: Perception paysanne de la variabilité climatique liée à la pluviométrie.

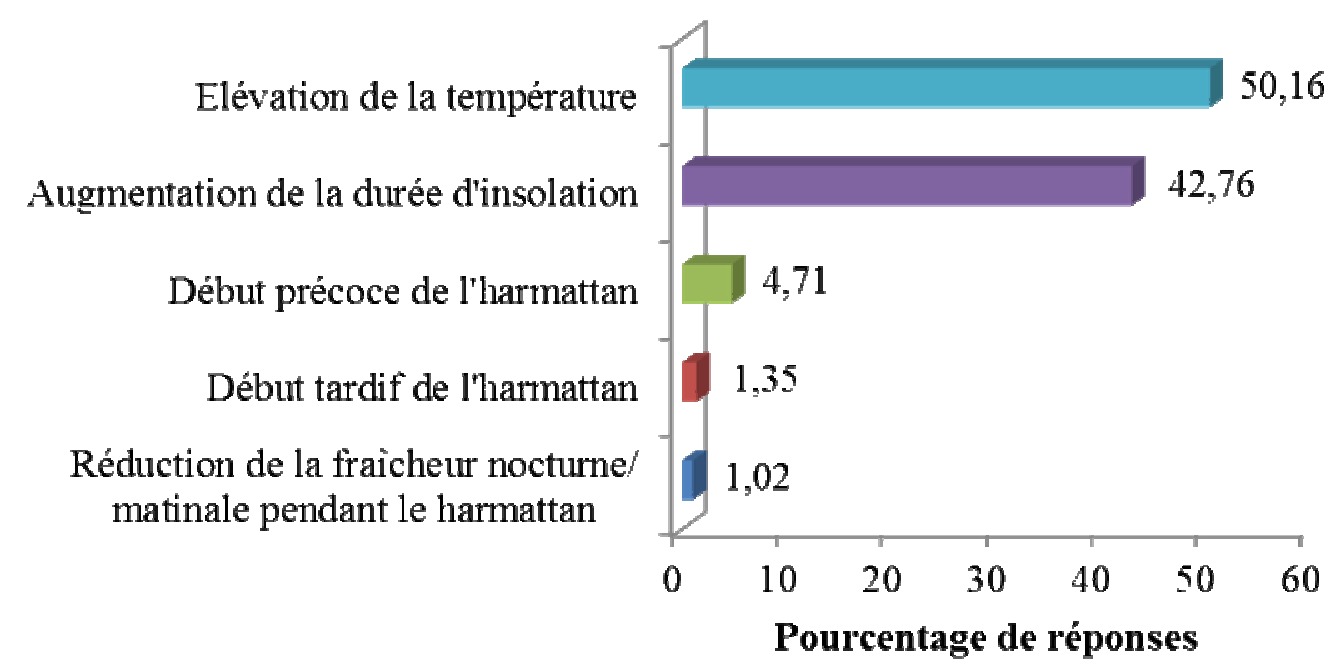

Figure 3: Perception paysanne de la variabilité climatique liée à la température. 


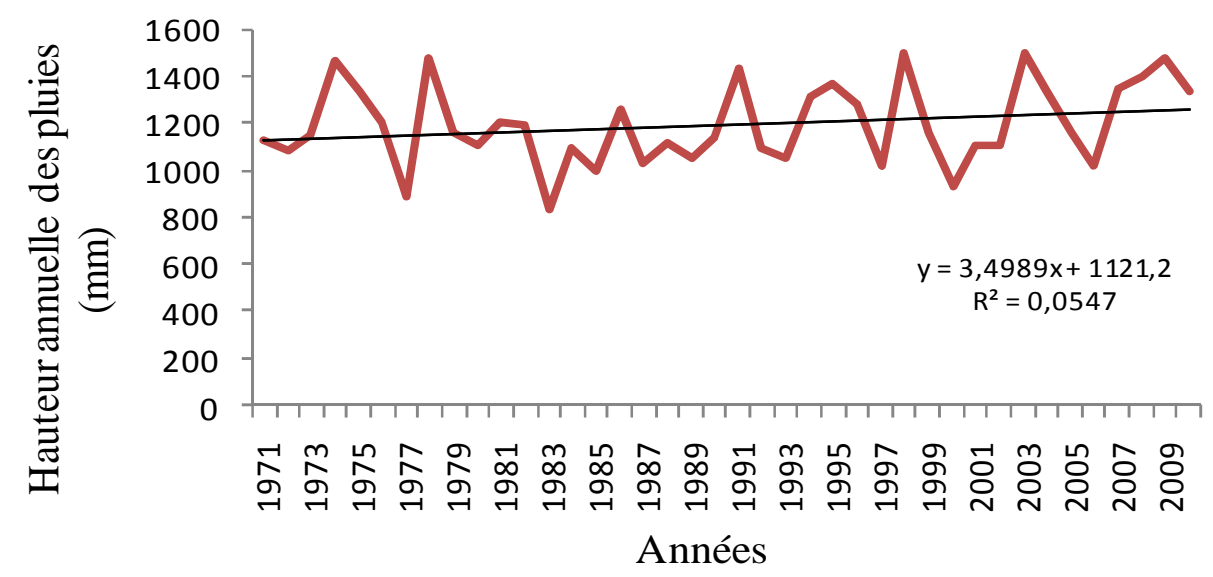

a) Evolution des données pluviométriques de la zone d'étude de 1971 - 2010.

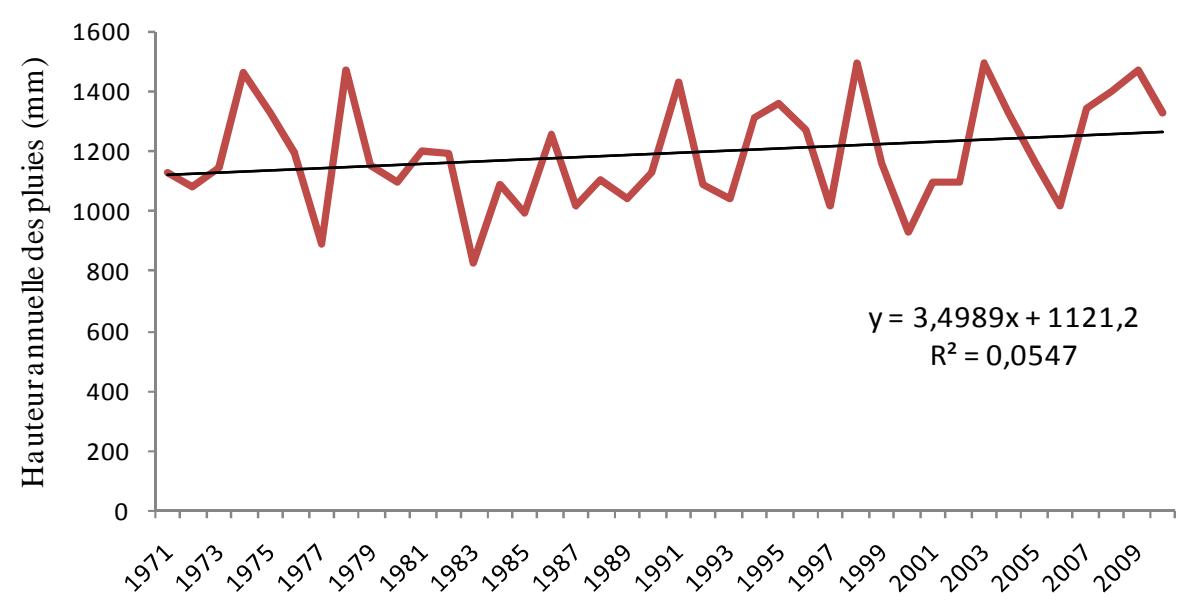

Années

b) Evolution des données pluviométriques de la zone d'étude de 1971 - 2010. 


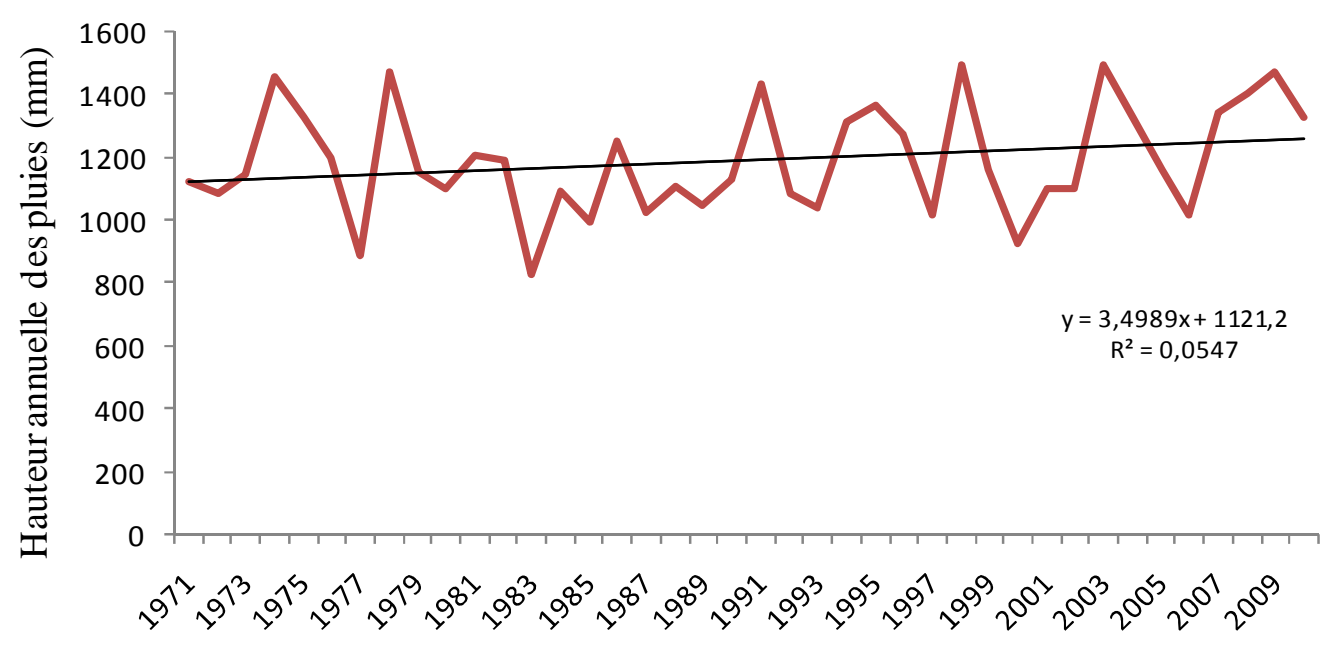

Années

c) Evolution des données pluviométriques de la zone d'étude de 1971 - 2010.

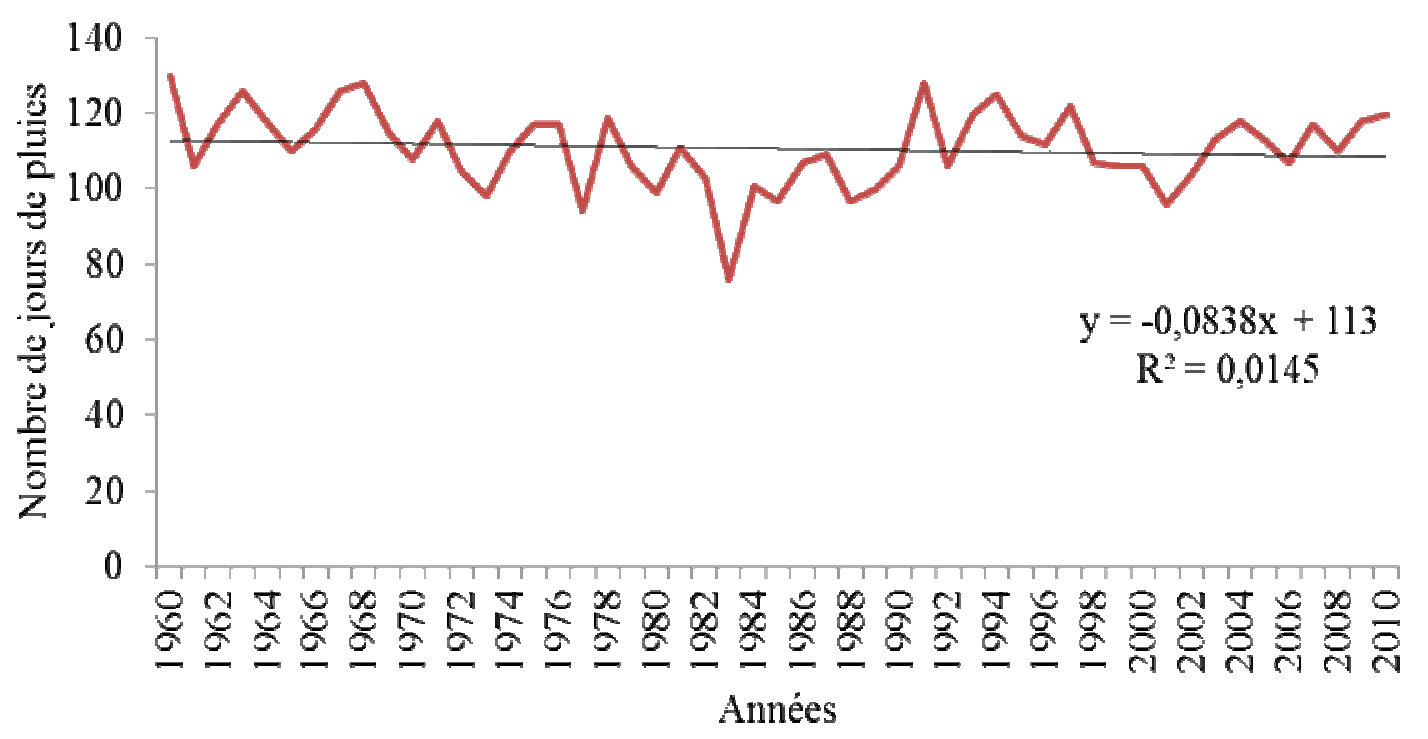

d) Evolution du nombre de jours de pluies dans la zone d'étude de 1960 - 2010.

Figure 4: Evolution des données pluviométriques dans la zone d'étude entre 1960 et 2010. 


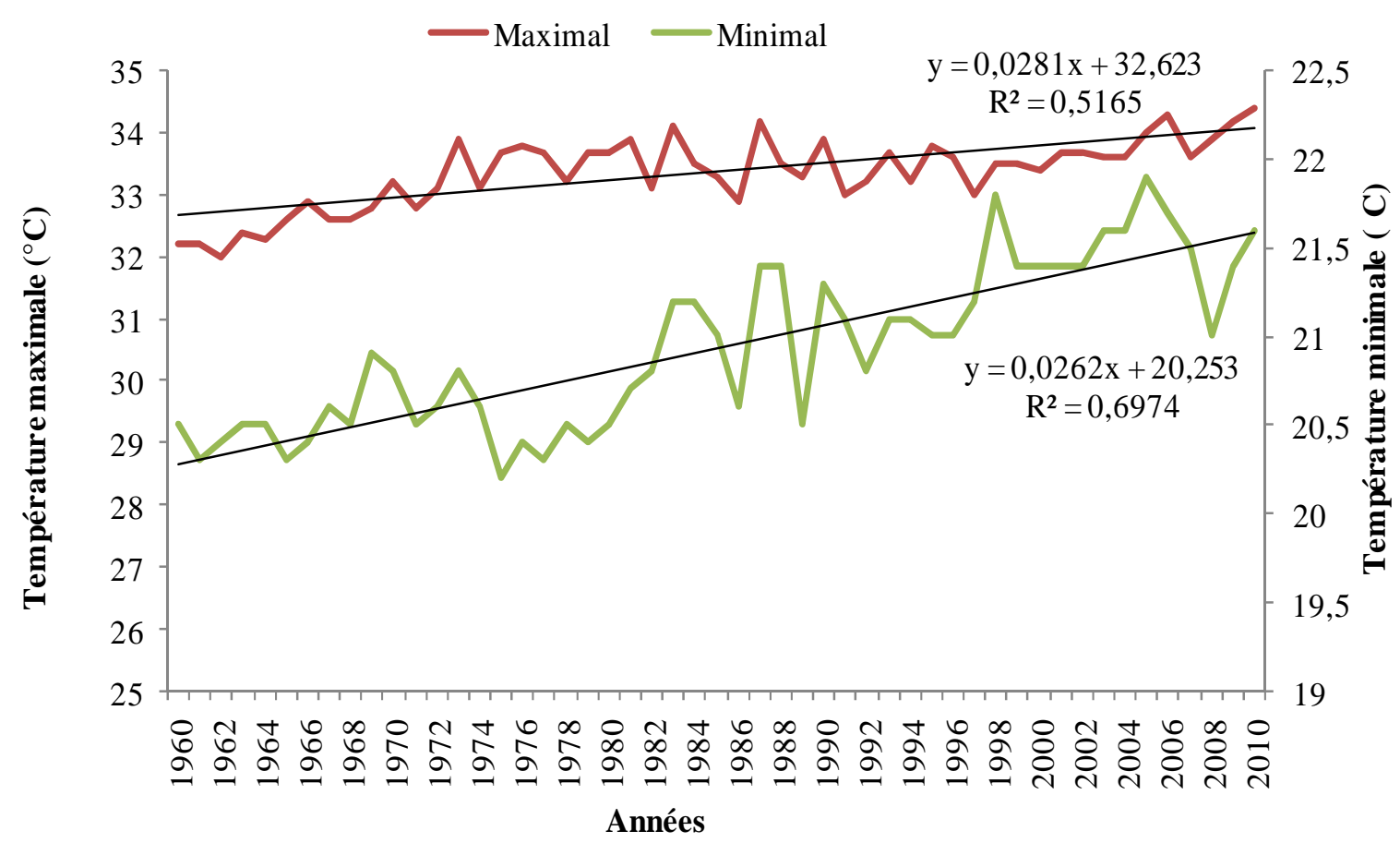

a) Evolution annuelle de la température dans la zone d'étude. 


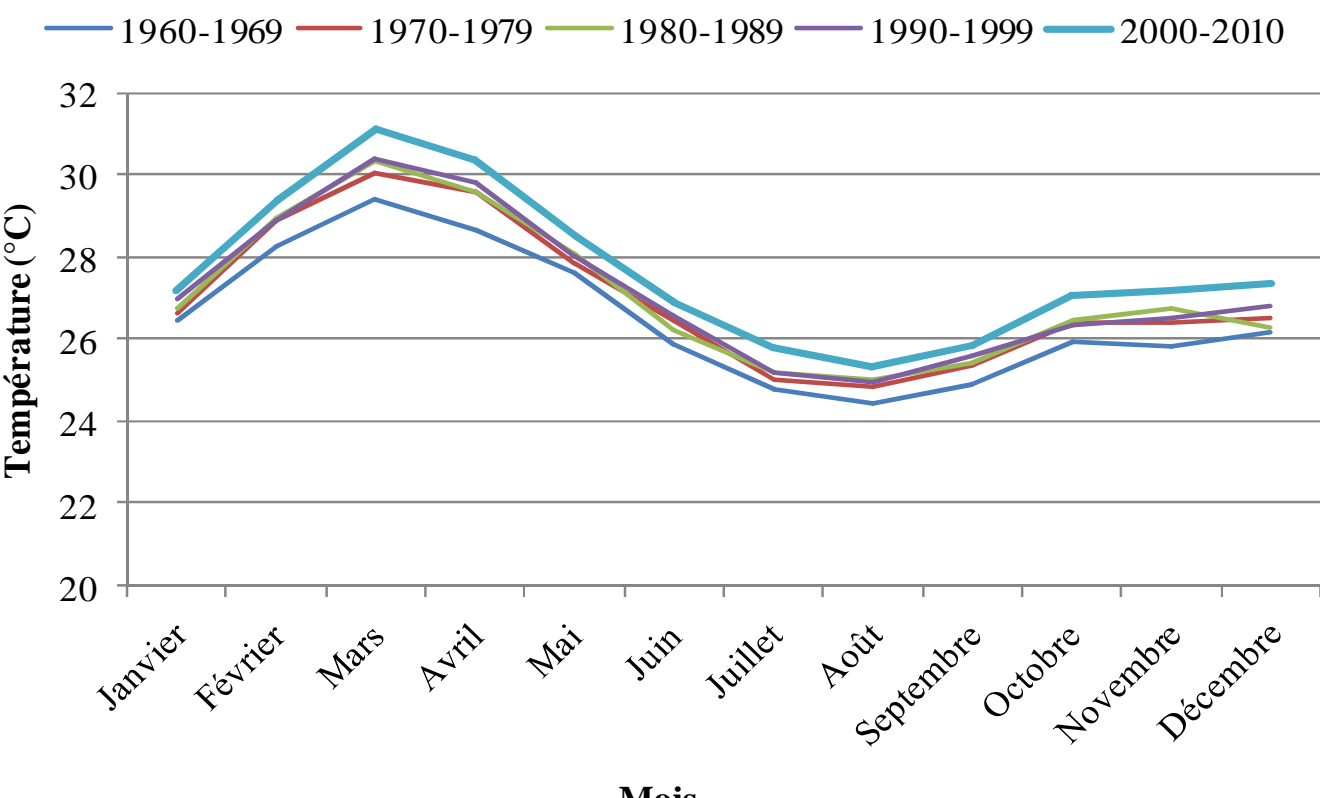

b) Distribution intra-annuelle de la température dans la zone d'étude.

Figure 5: Evolution de la température dans la zone d'étude entre 1960 à 2010. 
Y. L. LOKO et al. / Int. J. Biol. Chem. Sci. 7(2): 672-695, 2013

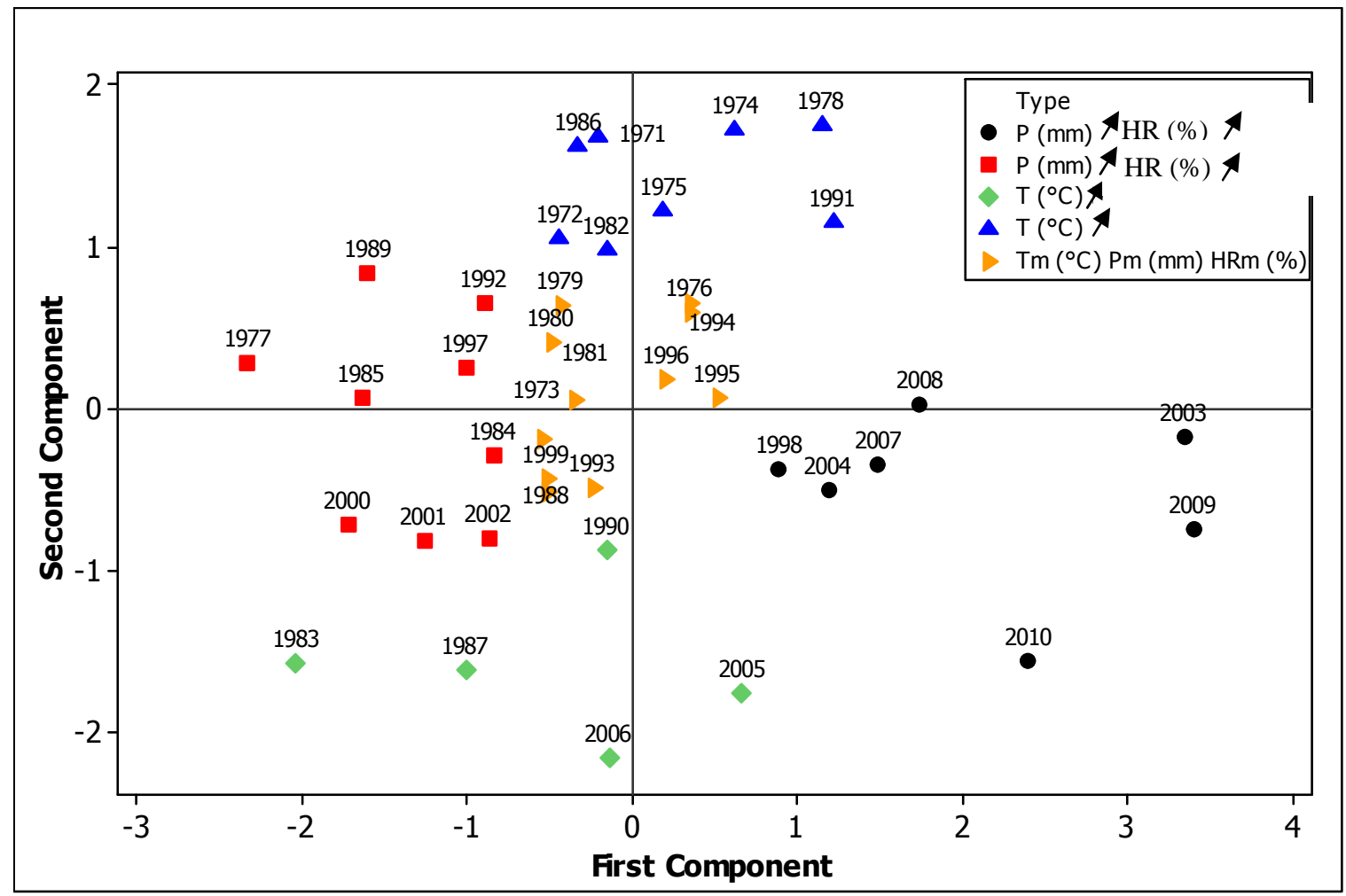

Figure 6 : Diagramme montrant des précipitations annuelles $(\mathrm{P})$, température $(\mathrm{T})$ et humidité relative $(\mathrm{HR})$ de 1971 à 2010. 


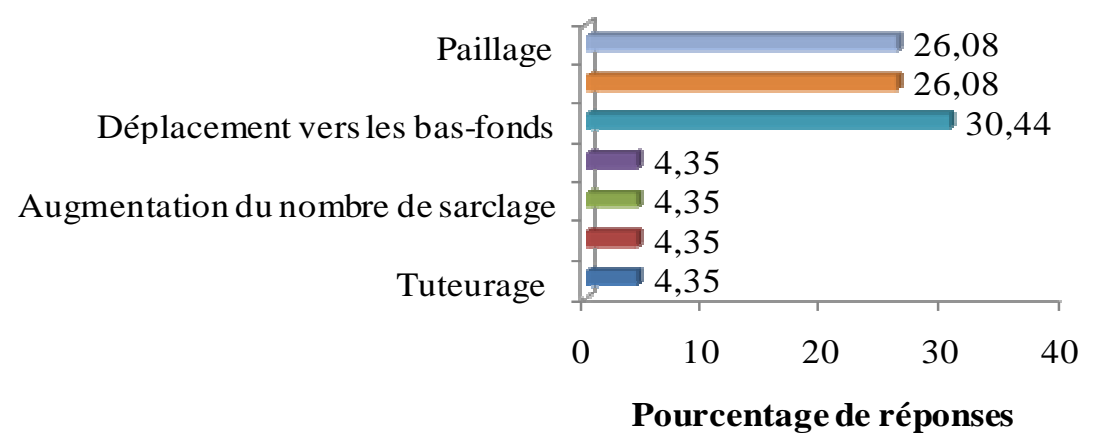

a) Stratégies d'adaptation en réponse à la sécheresse.

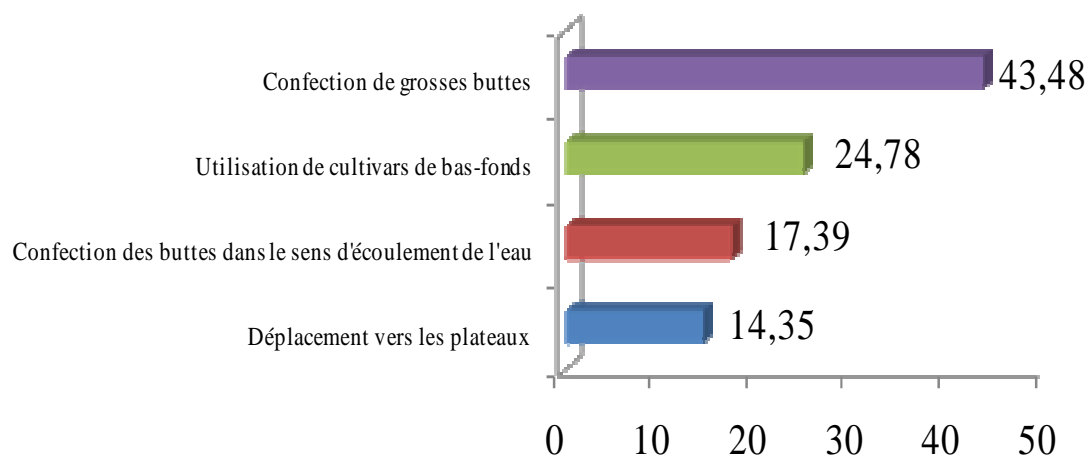

Pourcentage de réponses

b) Stratégies d'adaptation en réponse à l'inondation.

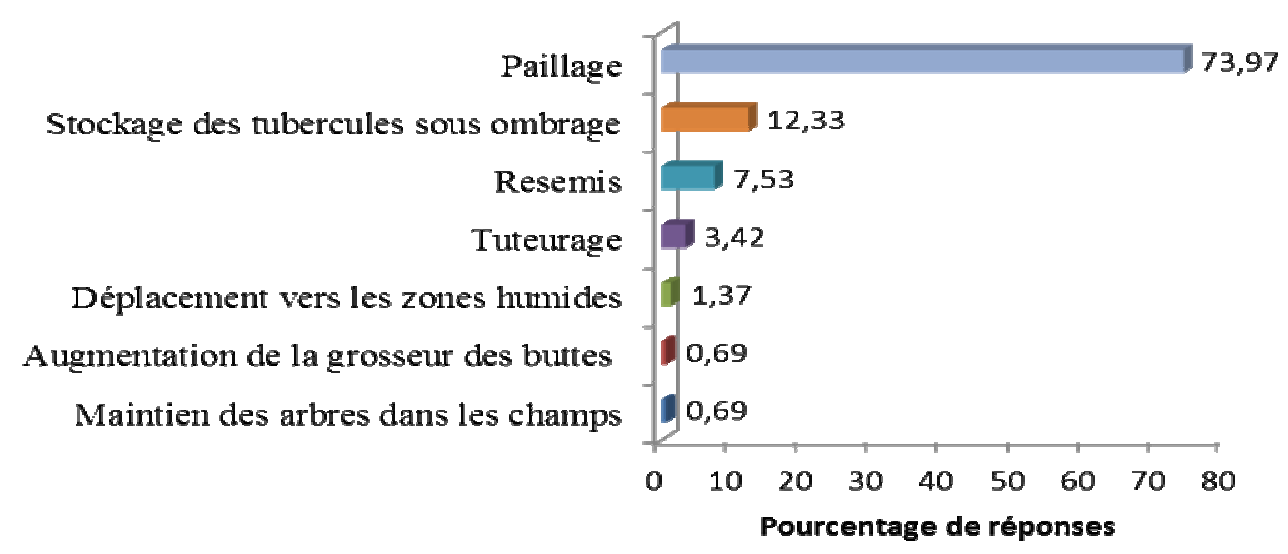

c) Stratégies d'adaptation en réponse à l'élévation de la température. 


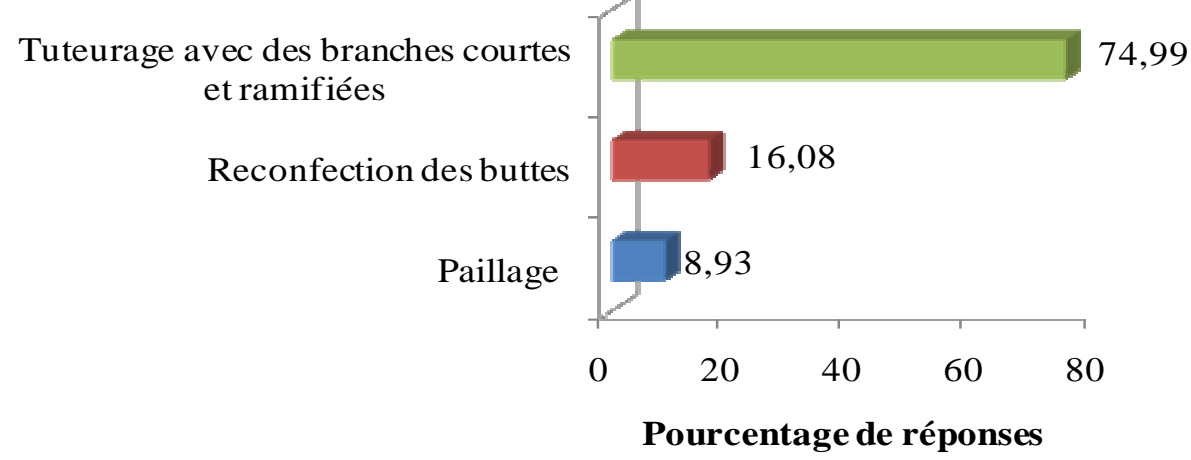

d) Stratégies d'adaptation en réponse aux vents violents.

Figure 7: Stratégies d'adaptation développées par les producteurs face aux changements climatiques.

Tableau 1: Relations entre les variables explicatives et les perceptions paysannes des changements climatiques révélées par l'analyse de la régression logique.

\begin{tabular}{lccc}
\hline Variables explicatives & Coefficients & Erreur standard & P-value \\
\hline Age & $0,062^{* * *}$ & 0,025 & 0,015 \\
Education & $-0,412$ & 0,298 & 0,166 \\
Taille de l'exploitation & $-0,444$ & 0,429 & 0,300 \\
Année d'experience & $-0,003$ & 0,024 & 0,883 \\
Taille du ménage & 0,034 & 0.047 & 0.467 \\
Genre & $2,686^{* * *}$ & 0,856 & 0,002 \\
Main d'œuvre & $-0,002$ & 0,087 & 0,979 \\
Appartenance à une organisation & $-0,503$ & 0,511 & 0,325 \\
paysanne & $-4,351^{* * *}$ & 1,315 & 0,001 \\
Constante & $-81,328099$ & & \\
-2 log-vraisemblance & 150 & \\
Nombre d'observation & 30,95 & \\
LR Chi Square $(8)$ & 0,0001 & \\
Prob $>$ Chi Square & 0,1599 & \\
Pseudo R ${ }^{2}$ & & \\
\hline \multicolumn{2}{c}{$* * * * * *$ significatif respectivement à $1 \%, 5 \%$ et $10 \%$ de niveau de probabilité . }
\end{tabular}


Tableau 2 : Déterminants de l'adaptation aux changements climatiques par les producteurs.

\begin{tabular}{lccc}
\hline Variables explicatives & Coefficients & Erreur standard & P-value \\
\hline Age & $-0,075^{*}$ & 0,043 & 0,079 \\
Education & 0,176 & 0,294 & 0,549 \\
Taille de l'exploitation & 0,087 & 0,416 & 0,834 \\
Année d'experience & $0,060^{* * *}$ & 0,023 & 0,008 \\
Taille du ménage & $-0,015$ & 0,023 & 0,500 \\
Sexe & $-0,646$ & 0,825 & 0,433 \\
Main d'œuvre & 0,091 & 0,083 & 0,272 \\
Appartenance à une organisation paysanne & 0,444 & 0,513 & 0,386 \\
Constante & 0,791 & 1,222 & 0,518 \\
-2 log-vraisemblance & $-86,223071$ & & \\
LR Chi Square (8) & 14,84 & & \\
Prob > Chi Square & 0,0623 & & \\
Pseudo R ${ }^{2}$ & 0,0792 & & \\
\hline$* * *, * *, *=$ significatif à 1\%, 5\% et 10\% de niveau de probabilité respectivement. &
\end{tabular}

\section{DISCUSSION}

L'étude a révélé que les producteurs d'igname du Nord-Ouest du Benin ont une bonne connaissance des variabilités climatiques. Des études menées dans de nombreux pays en Afrique (Mustapha et al., 2012) et dans le monde (Macharia et al., 2012) avaient déjà conduit à des résultats similaires. Les variabilités climatiques majeures signalées par les producteurs enquêtés ont été l'augmentation de la température et la diminution de la quantité des pluies. Ces mêmes perceptions paysannes ont été signalées par des études similaires effectuées au Zimbabwe (Moyo et al., 2012), au Zambie (Nyanga et al., 2011), en Uganda (Kisauzi et al., 2012), au Nigéria (Ugwoke et al., 2012), au Ghana (MacCarthy et Vlek, 2012) et même dans les régions du Sud Benin (Yabi et Afouda, 2012). Cependant, l'augmentation de la pluviométrie dans cette zone aride révélée par l'analyse des données météorologiques est surprenante et, à notre connaissance, n'a pas encore été signalée dans un autre pays. Sa combinaison avec la réduction du nombre de jours de pluie permet de comprendre les nombreux cas d'inondation signalés dans cette zone ces cinq dernières années.
Vue les forts taux de concordances obtenus entre la perception paysanne des années dominées par des conditions climatiques extrêmes et l'analyse des données météorologiques, il découle que les communautés locales ont une bonne mémoire des évènements climatiques passés comme ce fut le cas des communautés du sahel (Tazeze et al., 2012). En effet, comme signalé par les producteurs, Yabi et Afouda (2012) ont notés que les décennies 1970 et 1980 ont présentées des années ayant un déficit pluviométrique important (1973, 1977, 1982, 1983, 1984) autant dans la zone d'étude qu'à l'échelle du pays. Cependant, la mémoire des producteurs s'est portée sur des événements climatiques plus récents (à partir de 1982). Ceci n'est pas surprenant car plusieurs travaux (Deressa et al., 2011 ; Tazeze et al., 2012) ont montré des résultats similaires.

Yabi et Afouda (2012) ont indiqué que les changements climatiques sont perçus et compris de façons diverses par les populations africaines selon leurs caractéristiques socioculturelles. Sur cette base, la détermination des facteurs qui influencent la perception des producteurs peut, dans une certaine mesure, améliorer les politiques à mettre en œuvre pour faire face aux impacts négatifs des changements climatiques. 
Tableau 3: Déterminants du choix d'un producteur d'igname à utiliser une méthode spécifique d'adaptation au changement climatique.

\begin{tabular}{|c|c|c|c|c|c|c|c|c|c|c|}
\hline \multirow[t]{2}{*}{ Variables explicatives } & \multicolumn{2}{|c|}{$\begin{array}{c}\text { Utilisation de la } \\
\text { diversité variétale }\end{array}$} & \multicolumn{2}{|c|}{$\begin{array}{c}\text { Bonnes pratiques } \\
\text { culturales }\end{array}$} & \multicolumn{2}{|c|}{ Drainage } & \multicolumn{2}{|c|}{$\begin{array}{c}\text { Changement de } \\
\text { terrain }\end{array}$} & \multicolumn{2}{|c|}{ Multiples stratégies } \\
\hline & Coefficient & P-value & Coefficient & P-value & Coefficient & P-value & Coefficient & P-value & Coefficient & P-value \\
\hline Age & $-0,012$ & 0,728 & $-0,056$ & 0,132 & $-0,113$ & 0,174 & $-0,031$ & 0,412 & $-0,086^{* *}$ & 0,017 \\
\hline Sexe & $18,707 * * *$ & 0,000 & $-1,417$ & 0,143 & 17,900 & 0,203 & $-0,582$ & 0,658 & $-1,686^{*}$ & 0,081 \\
\hline Taille du ménage & $-0,030$ & 0,608 & $-0,103$ & 0,163 & $-0,190$ & 0,911 & $-0,010$ & 0,863 & $-0,052$ & 0,423 \\
\hline Main d'œuvre & 0,025 & 0,833 & $0,244 * *$ & 0,037 & 0,029 & 0,308 & $-0,114$ & 0,450 & 0,053 & 0,668 \\
\hline Expérience & 0,028 & 0,354 & $0,063^{*}$ & 0,067 & 0,766 & 0,787 & 0,034 & 0,332 & $0,075^{* *}$ & 0,025 \\
\hline Superficie emblavée & 0,661 & 0,223 & $-0,680$ & 0,357 & $-0,925$ & 0,541 & 0,888 & 0,143 & $-0,874$ & 0,212 \\
\hline Education & 0,088 & 0,843 & $-0,619$ & 0,206 & 0,665 & 0,329 & $1,062 * *$ & 0,017 & $-0,127$ & 0,757 \\
\hline Association & 0,405 & 0,613 & $1,288 *$ & 0,056 & $-38,35$ & 1.000 & 0,356 & 0,653 & 0,045 & 0,948 \\
\hline Constante & $-20,266$ & & 1,824 & 0,281 & $-17,51$ & & $-0,806$ & 0,697 & $3,893 * *$ & 0,018 \\
\hline Catégorie de base & \multicolumn{10}{|c|}{ Aucune adaptation } \\
\hline Nombre d'observation & 150 & & & & & & & & & \\
\hline LR Chi 2 (40) & 59,30 & & & & & & & & & \\
\hline-2 log-vraisemblance & - 199,39001 & & & & & & & & & \\
\hline Prob $>$ Chi 2 & 0,0252 & & & & & & & & & \\
\hline Pseudo $\mathrm{R}^{2}$ & 0,1295 & & & & & & & & & \\
\hline
\end{tabular}


Tableau 4: Critères de préférences paysans des cultivars d'igname dans la zone d'étude et à travers les aires ethniques.

\begin{tabular}{|c|c|c|c|c|c|c|}
\hline \multirow{2}{*}{ Critères de préférences } & \multirow{2}{*}{$\begin{array}{c}\begin{array}{c}\text { Zone d'étude } \\
\text { (\% de } \\
\text { réponses) }\end{array} \\
\end{array}$} & \multicolumn{5}{|c|}{ Importance par aire ethnique (\% de réponses) } \\
\hline & & Berba & Ditamari & Warma & Bialli & Natimba \\
\hline \multicolumn{7}{|l|}{ Critères environnementaux } \\
\hline Résistance à la sécheresse & 19,1 & 9,77 & 23,24 & 31,6 & 29,86 & 11,19 \\
\hline Tolérance à l'excès d'eau & 1,59 & 0 & 0 & 0 & 0 & 11,94 \\
\hline Adaptabilité à tout type de sol & 0,5 & 0 & 0 & 0,74 & 2,08 & 0 \\
\hline Tolérance aux sols pauvres & 2,99 & 5,17 & 1,06 & 4,46 & 4,17 & 0 \\
\hline Résistance aux ravageurs et maladies & 13,43 & 11,49 & 5,28 & 10,41 & 6,25 & 21,64 \\
\hline Résistance aux mauvaises herbes & 4,78 & 0 & 9,15 & 4,09 & 7,64 & 0 \\
\hline Total & 42,39 & 26,43 & 38,73 & 51,3 & 50 & 44,77 \\
\hline \multicolumn{7}{|l|}{ Critères agronomiques } \\
\hline Bonne conservation post-récolte & 12,64 & 14,94 & 13,38 & 13,01 & 9,03 & 13,43 \\
\hline Forte productivité & 8,46 & 12,07 & 13,03 & 3,72 & 3,47 & 8,96 \\
\hline Précocité de la variété & 5,77 & 7,47 & 4,58 & 6,32 & 4,17 & 6,72 \\
\hline Bonne forme et aspect de l'igname & 2,99 & 0 & 4,58 & 0 & 0 & 12,69 \\
\hline Non exigence en tuteurage & 1,29 & 0 & 0 & 0 & 6,94 & 0 \\
\hline Total & 31,15 & 34,48 & 35,57 & 23,05 & 23,61 & 41,8 \\
\hline \multicolumn{7}{|l|}{ Critères technologiques et culinaires } \\
\hline Bonne qualité d'igname pilée & 11,24 & 14,37 & 8,1 & 11,52 & 21,53 & 2,24 \\
\hline Bonne qualité d'igname bouillie & 1,99 & 5,75 & 3,52 & 0 & 0 & 0 \\
\hline Bonne qualité des frites d'igname & 2,99 & 4,02 & 5,63 & 2,6 & 0 & 0 \\
\hline Bonne qualité des cossettes & 5,57 & 10,34 & 3,87 & 3,72 & 4,86 & 7,46 \\
\hline Total & 21,79 & 34,48 & 21,12 & 17,84 & 26,39 & 9,7 \\
\hline \multicolumn{7}{|l|}{ Critère économique } \\
\hline Forte valeur marchande & 4,67 & 4,61 & 4,58 & 7,81 & $\mathbf{0}$ & 3,73 \\
\hline
\end{tabular}

Le fait que l'âge et le sexe du producteur ont été des facteurs déterminants la perception des changements climatiques n'est pas surprenant car Oyekale et Oladele (2012) ont montré qu'avec l'âge l'habilité à percevoir les changements climatiques augmentait et que chez les communautés rurales africaines les hommes perçoivent mieux les changements climatiques, probablement du fait de leur contact régulier avec l'extérieur et leurs meilleur accès aux sources d'information tel que la radio.
Selon les producteurs, les variabilités climatiques marquées surtout par des périodes de sécheresse et d'inondation ont des impacts négatifs sur la production et la diversité variétale de l'igname. Ce résultat était attendu puisque le régime pluviométrique est le facteur climatique le plus important qui influence les activités agricoles (Ayanlade et al., 2010). La baisse de productivité signalée par les producteurs comme principal impact des changements climatiques sur la culture de l'igname a été aussi indiquée sur d'autres 
cultures telles que le niébé (Ajetomobi et Abiodun, 2010), le riz (Nwalieji et Uzuegbunam, 2012) et le sorgho (MacCarthy et Vlek, 2012). Il en est de même pour la prolifération des insectes qui a été signalé comme impact des changements climatiques sur le sésame (Luka et Yahaya, 2012). La pourriture des tubercules d'igname dans les buttes sous l'effet de la chaleur ou d'un excès d'eau a été un impact négatif important signalé par les producteurs et qui se justifie car la température du sol affecte le développement des plantes plus que tout autre facteur et les précipitations excessives rendent de nombreuses cultures vulnérables à l'engorgement (Uguru et al., 2011). La dégradation et la baisse de la fertilité des sols contribuent aussi énormément à la chute de la production (Luka et Yahaya, 2012). Tous ces facteurs contribuent à une perte importante de la diversité variétale de l'igname et la réduction des superficies emblavées par les producteurs comme c'est le cas avec le sorgho au Ghana (MacCarthy et Vlek, 2012). Il urge donc de développer des stratégies complémentaires de conservation in situ et ex situ (MacCarthy et Vlek, 2012) et de concevoir des stratégies d'adaptations efficaces.

Bien que la plupart des producteurs interviewés aient noté des effets négatifs des changements climatiques sur la production d'igname, nombreux parmi eux $(39,30 \%)$ n'ont développés aucune stratégie adaptative. Ce résultat se rapproche de ceux obtenus par Nyanga et al. (2011) en Zambie, Tazeze et al. (2012) en Ethiopie et Luka et Yahaya (2012) au Nigeria. Selon Tidjani et Akponikpe (2012), les différentes pratiques d'adaptation notées sont soient, préventives ou curatives. Le paillage a des effets bénéfiques sur l'humidité et la température du sol et sur la croissance et le rendement de l'igname (Inyang, 2005). Le tuteurage, permet une meilleure exposition des feuilles d'igname ce qui augmente la capacité d'absorption de la lumière et de l'humidité de l'air et donc une amélioration de la productivité (Sadauki et
Olanrewaju, 2012). Les grosses buttes assurent une bonne fraîcheur aux tubercules semences et les préservent contre les pourritures (Srivastava et al., 2012). Par ailleurs, pour pallier à la sécheresse, les producteurs abandonnent les cultivars à cycle long (igname tardive à une seule récolte) au profit des cultivars à cycle court (igname précoces à deux récoltes). Cette stratégie est aussi utilisée par les producteurs pour d'autres cultures telles que le niébé (Baco et al., 2008) et le maïs (Tidjani et Akponikpe, 2012). La dominance des ignames précoce dans les zones arides de production d'igname a été déjà signalée par Loko et al. (2013) au Bénin et Dansi et al. (2013) au Togo. Malgré toutes ces méthodes et selon Dansi et al. (2013), l'utilisation de cultivars d'igname résistants à la sécheresse et / ou à l'excès d'humidité du sol semble être la stratégie la plus adaptée à la production de l'igname dans le contexte des changements climatiques. Malheureusement, très peu de cultivars sont résistants à la sécheresse ou à l'excès d'humidité du sol (ignames de bas-fonds). Il urge donc de renforcer la diversité variétale dans la zone d'étude par des introductions à partir de la collection nationale (Loko et al. 2013) ou par la création variétale en prenant en compte des critères paysans de préférences variétale identifiés et hiérarchisés.

Deux principaux facteurs (âge et expérience) influencent l'aptitude des producteurs à adapter leur production d'igname aux changements climatiques dans la zone d'étude. L'âge du producteur influence négativement l'adaptation aux changements climatiques et particulièrement l'utilisation de multiples stratégies par les producteurs. Ces résultats sont contraires à ceux obtenus par Tazeze et al. (2012) en Ethiopie mais conformes à ceux rapportés par Oyekale et Oladele (2012) au Nigéria. Quant à l'expérience du producteur, les résultats ont montré une influence positive sur l'adaptation aux changements climatiques et particulièrement sur l'adoption de bonnes pratiques agricoles. Ces résultats sont en 
adéquation avec ceux de Falola et al. (2012) et peuvent s'expliquer par le fait que les agriculteurs acquièrent et développent plus de compétences au fil du temps et ceci leur permet de prendre des mesures adaptatives contre l'impact des changements climatiques. Le genre (homme, femme) n'a pas eu d'effet significatif sur l'adaptation au changement climatique mais influence de façon significative le type de stratégie adoptée par les producteurs tel que l'utilisation de la diversité variétale et de stratégies multiples. Des résultats similaires ont été obtenus par Falola et al. (2012). Cette sensibilité d'adaptation des hommes à l'utilisation de la diversité variétale peut être expliqué par le fait que la culture de l'igname est une activité exclusivement réservée aux hommes et l'implication des femmes ne se fait qu'après la récolte dans les transformations alimentaires et la commercialisation sur le marché (Baco et al., 2008). Des cas similaires ont été signalés sur le fonio (Digitaria exilis) au Nord-Ouest du Bénin (Dansi et al., 2010). Le niveau d'éducation $\mathrm{du}$ producteur a influencé significativement la migration des champs des plateaux vers les bas-fonds et inversement. L'étude de Deressa et al. (2011) a donné des résultats similaires et révèle que le niveau d'éducation du producteur augmente la probabilité d'adaptation aux changements climatiques. L'appartenance à une association de producteurs a influé positivement sur le choix du producteur à utiliser de bonnes pratiques agricoles. Les associations paysannes étant connues comme des centres d'échange d'idées et de partage d'information entre producteurs, une participation régulière aux réunions se traduirait donc par une probabilité accrue d'adaptation aux changements climatiques.

\section{Conclusion}

La présente étude a révélé que les producteurs de la zone de l'Atakora au nordouest du Bénin sont bien conscients des variabilités climatiques que subissent leur zone et ont une bonne perception aussi bien de leurs effets (augmentation de la température, diminution de la pluviométrie, sécheresse, excès de pluie et inondations occasionnelles, etc.) que de leurs impacts sur la production de l'igname. Bien que l'étude ait révélé, entre autre critères, que c'est surtout les hommes et les personnes âgées qui font plus appel aux stratégies d'adaptation, les femmes et les jeunes ne doivent être en aucun cas exclus des politiques nationales de réduction des impacts liés aux changements climatiques. Sur la base des résultats de l'étude, nous recommandons la sensibilisation sur les changements climatiques et la diffusion de cultivars précoces, très productifs et résistants à la sécheresse et à l'excès d'humidité. Ceux-ci existent dans l'agriculture traditionnelle et il faut les identifier par des évaluations participatives à travers toutes les zones de production au Bénin.

\section{REFERENCES}

Baco MN, Ahanchedé A, Bello S, Dansi A, Vodouhè R, Biaou G, Lescure JP. 2008. Evaluation des pratiques de gestion de la diversité du niébé (Vigna unguiculata): une tentative méthodologique expérimentée au Bénin. Cahiers Agric., 17: 183-188.

Dansi A, Dantsey-Barry H, Dossou-Aminon I, N'Kpenu EK, Agré AP, Sunu YD, Kombaté K, Loko YL, Dansi M, Assogba P, Vodouhè R. 2013. Varietal diversity and genetic erosion of cultivated yams (Dioscorea cayenensis Poir - D. rotundata Lam complex and $D$. alata L.) in Togo. International Journal of Biodiversity and Conservation (In press).

Mignouna HD, Dansi A. 2003. Yam (Dioscorea sp.) domestication by the Nago and Fon ethnic groups in Benin. Genetic Resources and Crop Evolution, 50(5): 519-528.

Adesiji GB, Matanmi BM, Onikoyi MP and Saka MA. 2012. Farmers' perception of climate change in Kwara State, Nigeria. World Rural Observations, 4(2): 46-54.

Ajetomobi J, Abiodun A. 2010. Climate change impacts on cowpea productivity 
in Nigeria. African Journal of Food Agriculture Nutrition and Development, 10(3): 2258-2217.

Ayanlade A, Odekunle TO, Orimoogunje OOI. 2010. Impacts of climate variability on tuber crops in Guinea Savanna Part of Nigeria: A GIS Approach. Journal of Geography and Geology, 2(1): 28-35.

Dansi A, Adoukonou-Sagbadja H, Vodouhè R. 2010. Diversity, conservation and related wild species of Fonio millet (Digitaria spp) in the northwest of Benin. Genet. Resour. Crop Evol., 57: 827-839.

Deressa TT, Hassan RM, Ringler C. 2011. Perception of and adaptation to climate change by farmers in the Nile basin of Ethiopia. Journal of Agricultural Science, 149: 23-31.

Falola A, Fakayode SB, Akangbe JA, Ibrahim HK. 2012. Climate change mitigation activities and determinants in the rural Guinea Savannah of Nigeria. Sustainable Agriculture Research, 1(2):170-178.

Inyang EU. 2005. An evaluation of tillage and storage systems applied by traditional root crop farmers in Cameroon. Agric. Envron. J., 7(2): 15-22.

Kisauzi T, Mangheni MN, Seguya H, Bashaasha B. 2012. Gender dimensions of farmers' perceptions and knowledge on climate change in Teso sub-region, eastern Uganda. African Crop Science Journal, 20(2): 275 - 286.

Kombo GR, Dansi A, Loko LY, Orkwor GC, Vodouhè R, Assogba $\mathrm{P}$, Magema JM. 2012. Diversity of cassava (Manihot esculenta Crantz) cultivars and its management in the department of Bouenza in the Republic of Congo. Genetic Resources and Crop Evolution, 59(8): 1789-1803.

Labintan AC, Ding S. 2012. An assessment of agricultural productivity and major driving factors in the republic of Benin. Ethiopian Journal of Environmental Studies and Management, 5(4): 470-476.

Loko YL, Dansi A, Linsoussi C, Tamo M, Vodouhè R, Akoegninou A, Sanni A.
2013. Current status and spatial analysis of Guinea yam (Dioscorea cayenensis Lam. -D. rotundata Poir. complex) diversity in Benin. Genetic Resources and Crop Evolution (in press).

Luka EG, Yahaya H. 2012. Sources of awareness and perception of the effects of climate change among sesame producers in the southern agricultural zone of Nasarawa State, Nigeria. Journal of Agricultural Extension, 16(2): 134-143.

MacCarthy DS, Vlek PLG. 2012. Impact of climate change on sorghum production under different nutrient and crop residue management in semi-arid region of Ghana: a modeling perspective. African Crop Science Journal, 20(2): 243 - 259.

Macharia PN, Thuranira EG, Nganga LW, Lugadiru J, Wakori S. 2012. Perceptions and adaptation to climate change and variability by immigrant farmers in semiarid regions of Kenya. African Crop Science Journal, 20(1): 287-296.

Moyo M, Mvumi BM, Kunzekweguta M, Mazvimavi K, Craufurd P, Dorward P. 2012. Farmer perceptions on climate change and variability in semi-arid Zimbabwe in relation to climatology evidence. African Crop Science Journal, 20(2): 317 - 335 .

Mustapha SB, Sanda AH, Shehu H. 2012. Farmers' perception of climate change in central agricultural zone of Borno State, Nigeria. Journal of Environment and Earth Science, 2(11): 21-27.

Nabikolo D, Bashaasha B, Mangheni MN, Majaliwa JGM. 2012. Determinants of climate change adaptation among male and female headed farm households in eastern Uganda. African Crop Science Journal, 20(2): 203 - 212.

Naess LO. 2013. The role of local knowledge in adaptation to climate change. Wiley Interdisciplinary Reviews: Climate Change, 4: 99-106.

Nwalieji HU, Uzuegbunam CO. 2012. Effect of climate change on rice production in 
Anambra State, Nigeria. Journal of Agricultural Extension. 16(2): 81-91.

Nyanga H, Johnsen FH, Aune JB. 2011. smallholder farmers' perceptions of climate change and conservation agriculture: evidence from Zambia. Journal of Sustainable Development, 4(4): 73-85.

Oyekale AS, Oladele OI. 2012. Determinants of climate change adaption among cocoa farmers in southwest Nigeria. Journal of Food, Agriculture \& Environment, 10(34): 1562-1567.

Sadauki AA, Olanrewaju RM. (2012). Effect of climate on yam tuberization in the guinea savanna ecological zone of Nigeria: the case study of Kwara State. Ethiopian Journal of Environmental Studies and Management, 5(2): 138-146.

Srivastava AK, Gaisera T, Paethb H, Ewertc F. 2012. The impact of climate change on Yam (Dioscorea alata) yield in the savanna zone of West Africa. Agriculture Ecosystems \& Environment, 153: 57-64.

Tazeze A, Haji J, Ketema M. 2012. Climate change adaptation strategies of smallholder farmers: the case of Babilie
District, East Harerghe zone of Oromia Regional State of Ethiopia. Journal of Economics and Sustainable Development, 3(14): 1-12.

Tidjani MA, Akponikpe PBI. 2012. Évaluation des stratégies paysannes d'adaptation aux changements climatiques : cas de la production du maïs au Nord-Bénin. African Crop Science Journal, 20(2): 425 - 441.

Uguru MI, Baiyeri KP, Aba SC. 2011. Indicators of climate change in the derived savannah niche of Nsukka, South-eastern Nigeria. Journal of Tropical Agriculture, Food, Environment and Extension, 10(1): 17 -26.

Ugwoke FO, Nnadi FN, Anaeto CF, Aja OO, Nwakwasi RN. 2012. Crop Farmers' Perception of and Adaptation to Climate Change in Orlu Agricultural Zone of Imo State, Nigeria. Journal of Agricultural Extension, 16(2): 212-224.

Yabi I, Afouda F. 2012. Extreme rainfall years in Benin (West Africa). Quaternary International, 262(7): 39-43. 